\title{
Relação entre função sensório-motora e equilíbrio funcional de indivíduos crônicos após acidente vascular cerebral
}

\author{
Relationship between the sensory-motor function and functional balance of chronic \\ individuals after stroke
}

\author{
Matheus de Sales Santos ${ }^{1}$, Nildo Manoel da Silva Ribeiro ${ }^{2 *}$ \\ ${ }^{1}$ Mestrando da Pós-Graduação de Processos Interativos dos Órgãos e Sistemas, Instituto de Ciências da Saúde, \\ UFBA; ${ }^{2}$ Doutor em Neurologia/Neurociências pela UNIFESP. \\ Professor Adjunto do Departamento de Fisioterapia da UFBA.
}

\begin{abstract}
Resumo
Introdução: danos à função sensório-motora podem ocorrer após um acidente vascular cerebral (AVC), contribuindo para o surgimento de disfunções no controle do movimento, o que inclui a manutenção do controle postural. Déficits de equilíbrio são comuns em indivíduos após AVC, preditores de funcionalidade e integração comunitária. Portanto, é necessária a identificação de possíveis fatores associados a alterações do equilíbrio funcional desses indivíduos. Objetivo: analisar a associação entre função sensóriomotora e equilíbrio funcional de indivíduos hemiparéticos crônicos após AVC. Metodologia: estudo transversal, com indivíduos que sofreram AVC, de idade entre 18 e 80 anos, com hemiparesia por no mínimo 6 meses, capazes de deambular, sem déficit visual e(ou) auditivo, sem sequelas cognitivas e sem outros diagnósticos neurológicos e(ou) ortopédicos que limitem a mobilidade. A escala de Fugl-Meyer e a escala de equilíbrio de Berg foram usadas para avaliar a função sensório-motora e o equilíbrio funcional, respectivamente. Resultados: 63 indivíduos foram incluídos no estudo, com idade média de 56 anos e tempo mediano de AVC de 36 meses. Houve uma correlação positiva moderada com a sensibilidade $(r=0,512)$ e uma correlação que variou de fraca a desprezível com mobilidade $(r=0,489)$, dor $(r=-0,163)$, função motora de membro superior $(r=0,098)$ e função motora de membro inferior $(r=$ $0,344)$. Conclusão: déficits no equilíbrio funcional estiveram associados moderadamente às alterações na sensibilidade dos indivíduos hemiparéticos crônicos após AVC. Mobilidade, dor e função motora dos membros superiores e inferiores não demostraram uma associação importante com o equilíbrio funcional.
\end{abstract}

Palavras-chave: Equilíbrio postural. Paresia. Acidente vascular cerebral.

\begin{abstract}
Introduction: damages to the sensory-motor function can occur after a brain injury, contributing to the emergence of malfunctions in the control of the movement, including the maintenance of postural control. Balance deficits are common in subjects after stroke, and are predictors of community functionality and integration. Therefore, it is necessary to identify possible factors that are associated with changes in the functional balance of these individuals. Objective: to analyze the association between the sensory-motor function and functional balance of chronic hemiparetic individuals after stroke. Methodology: cross-sectional study, with post-stroke individuals between 18 and 80 years with hemiparesis for at least 6 months, able to walk, without visual and/or auditory deficit, without cognitive sequelae and without other neurological and/or orthopedic diagnosis that limit mobility. The Fugl-Meyer and Berg balance scale were used to evaluate the sensory-motor function and functional balance, respectively. Results: 63 individuals were included in the study, with an average age of 56 years and average time of stroke of 36 months. There was a moderate positive correlation with sensitivity ( $r=0.512)$ and a correlation between low to negligible with mobility $(r=0,489)$, pain $(r=-0,163)$, motor function of upper limb $(r=0,098)$ and motor function of lower limbs $(r=0,344)$. Conclusion: functional balance deficits were mildly associated with changes in sensitivity of chronic hemiparetic individuals post-stroke. Mobility, pain, motor function of upper limbs and lower limbs did not demonstrate a significant association with the functional balance.
\end{abstract}

Key-words: Postural balance. Paresis. Stroke.

\section{INTRODUÇÃO}

O acidente vascular cerebral (AVC) é a segunda maior causa de morte e a terceira maior causa de incapacidade no mundo (FEIGIN; NORRVING; MENSAH, 2017). As manifestações clínicas do AVC variam de acordo com a

Correspondente/Corresponding: *Nildo Manoel da Silva Ribeiro - Instituto de Ciências da Saúde, Universidade Federal da Bahia - End.: Av. Reitor Miguel Calmon, s/n, Vale do Canela, Salvador - BA. CEP: 40110100. Tel: (71) 3283-8910 - E-mail: nildo.ribeiro@ufba.br localização e extensão da lesão, o que torna o AVC heterogêneo no tipo, nos sinais, na gravidade e na recuperação. Suas principais consequências envolvem sequelas de percepção, cognitivas, perceptuais e sensório-motoras (STOKES, 2000).

Após a lesão cerebral, áreas responsáveis pela função sensório-motora podem estar danificadas, resultando em déficits sensoriais e (ou) motores nas extremidades contralaterais (NUDO; MCNEAL, 2013). Para uma recuperação efetiva do movimento após o AVC, deve existir uma 
relação otimizada entre as funções sensoriais e motoras (LAIBLE et al., 2012). Portanto, danos a essa inter-relação sensório-motora contribuem para o surgimento de disfunções do movimento e alterações no controle postural (HAART et al., 2004).

Déficits de equilíbrio são comuns em indivíduos após AVC, e podem estar associados a fraqueza muscular e a alterações na sensibilidade (TYSON et al., 2006), componentes da função sensório-motora. Estudos mostram que o equilíbrio funcional, relacionado à capacidade de o indivíduo se manter estável durante a realização de tarefas, é um preditor de desempenho nas atividades de vida diária (AVD) em indivíduos após AVC (CHO; LEE, 2013) e está diretamente associado ao nível de integração comunitária após AVC (KWONG et al., 2017). Portanto, é importante a identificação de possiveis fatores que estejam associados a alterações do equilíbrio funcional desses indivíduos e, consequentemente, a definição de possíveis alvos terapêuticos. Desse modo, o objetivo do estudo é analisar a associação entre a função sensoriomotora e equilíbrio funcional de indivíduos hemiparéticos após acidente vascular cerebral, na fase crônica.

\section{METODOLOGIA}

Trata-se de um estudo transversal, composto por indivíduos matriculados no Hospital Universitário Professor Edgard Santos em Salvador (BA), Brasil, no período de 2014 a 2018. O estudo foi aprovado pelo Comitê de Ética em Pesquisa do Instituto de Ciências da Saúde da Universidade Federal da Bahia, conforme o parecer consubstanciado $\mathrm{n}$ - 943.738. Todos os participantes assinaram o Termo de Consentimento Livre e Esclarecido, em consonância com os aspectos éticos previstos na Resolução 466/12 do Conselho Nacional de Saúde, respeitando-se os princípios da declaração de Helsinque.

Foram incluídos indivíduos entre 18 e 80 anos, com diagnóstico clínico neurológico de AVC, que apresentassem hemiparesia acima de 6 meses de início da doença, capacidade de deambular com ou sem dispositivo auxiliar de marcha e ausência de déficit visual ou auditivo (relatado pelo sujeito). Não foram incluídos indivíduos com sequelas cognitivas (Mini Exame do Estado Mental: escore $<24)$ e com outras patologias neurológicas e ortopédicas que prejudicassem a mobilidade.

Para avaliação da função sensório-motora, a Escala de Fugl-Meyer (EFM) foi aplicada. A EFM é um instrumento criado para mensurar a recuperação sensório-motora após AVC. É composta por um sistema de pontuação numérica acumulativa, que avalia seis aspectos do indivíduo: amplitude de movimento (ADM), dor, sensibilidade, função motora (FM) da extremidade superior e inferior e equilíbrio. Uma escala ordinal de três pontos é aplicada em cada item - não pode ser realizado (0); é realizado parcialmente (1); é realizado completamente (2) - totalizando 226 pontos (FUGL-MEYER et al., 1975; GLADSTONE; DANELLS; BLACK, 2002; MAKI et al., 2006).

Para uma função motora normal, a EFM possui um total de 100 pontos, com pontuação máxima de 66 para a extremidade superior e 34 para a extremidade inferior. $\mathrm{Na}$ avaliação motora, estão inclusas a mensuração do movimento, a coordenação e a atividade reflexa de ombro, cotovelo, punho, mão, quadril, joelho e tornozelo. Similarmente, há uma pontuação máxima de 24 para sensibilidade, 14 para equilíbrio, 44 para ADM e 44 para dor articular. Possui validação e excelente confiabilidade em indivíduos após AVC (FUGL-MEYER et al., 1975; GLADSTONE; DANELLS; BLACK, 2002; MAKI et al., 2006).

A escala de equilíbrio de Berg (EEB) é um instrumento criado para avaliação do equilíbrio funcional. É composta de 14 tarefas comuns no dia a dia, mensuradas de forma hierárquica, que avaliam o equilíbrio desde posições estáticas, como manter-se sentado, até tarefas dinâmicas, como manter-se em ortostase em apoio unipodal. Cada tarefa é pontuada de 0 a 4, com escore máximo de 56 pontos: quanto maior a pontuação, melhor o equilíbrio funcional. Ela possui tradução e confiabilidade para a população brasileira, além de ser apropriada e efetiva na avaliação do equilíbrio de indivíduos após AVC (BLUM; KORNER-BITENSKY, 2008; MIYAMOTO et al., 2004).

Trata-se de uma amostra não probabilística, por conveniência. Uma análise descritiva foi usada para apresentar as características sociodemográficas, clínicas e os achados do estudo, apresentada na forma de frequência absoluta e relativa para variáveis categóricas, e medidas de tendência central e dispersão para variáveis discretas e contínuas. Para a análise de correlação entre as variáveis equilíbrio funcional e função sensório-motora, e seus domínios, foi aplicado o teste de correlação de Pearson. A correlação foi qualificada da seguinte forma: 0,90 a 1.00 $(-0,90$ a - 1.00) - correlação positiva (negativa) muito forte; 0,70 a $0,90(-0,70$ a - 0,90) - correlação positiva (negativa) forte; 0,50 a $0,70(-0,50$ a - 0,70) - correlação positiva (negativa) moderada; 0,30 a $0,50(-0,30$ a $-0,50)$ - correlação positiva (negativa) fraca; 0,00 a $0,30(-0,00$ a $-0,30)$ - correlação positiva (negativa) desprezível (MUKAKA, 2012). Os dados foram analisados no pacote estatístico GraphPad Prism, versão 5.0.

\section{RESULTADOS}

Foram incluídos no estudo 63 indivíduos com idade média de 56 anos, sendo $51 \%$ homens, com tempo mediano de AVC de 36 meses. Dentre eles, 90,5\% sofreram AVC do tipo isquêmico, com o lado direito afetado em $52,4 \%$ dos casos. Houve ocorrência de quedas, nos últimos 6 meses, em $46 \%$ dos casos, e 49,2\% dos indivíduos utilizavam algum tipo de dispositivo auxiliar de marcha. 
As características clínicas e demográficas dos participantes estão apresentadas na tabela 1.

Tabela 1 - Características clínicas e demográficas dos 63 indivíduos após AVC crônico

\begin{tabular}{|c|c|c|c|c|}
\hline Variáveis & $N(63)$ & $\%$ & Média* (DP) & Mediana (IQ) \\
\hline \multicolumn{5}{|l|}{ Gênero } \\
\hline Homem & 31 & 49,2 & - & - \\
\hline Mulher & 32 & 50,8 & - & - \\
\hline Idade - anos & - & - & $56,1(10,2)$ & - \\
\hline Tempo de AVC-meses & - & - & - & $36(114,5)$ \\
\hline \multicolumn{5}{|l|}{ Tipo de AVC } \\
\hline Isquêmico & 57 & 90,5 & - & - \\
\hline Hemorrágico & 6 & 9,5 & - & - \\
\hline \multicolumn{5}{|l|}{ Lado acometido } \\
\hline Direito & 33 & 52,4 & - & - \\
\hline Esquerdo & 30 & 47,6 & - & - \\
\hline $\begin{array}{l}\text { Ocorrência de quedas } \\
\text { em } \leq 6 \text { meses }\end{array}$ & 29 & 46 & - & - \\
\hline $\begin{array}{l}\text { Uso de dispositivo } \\
\text { auxiliar de marcha }\end{array}$ & 31 & 49,2 & - & - \\
\hline
\end{tabular}

\% = Frequência relativa; DP = Desvio padrão; $\mathrm{N}=$ Número de sujeitos; IQ = Intervalo interquartílico.

* = aritmética.

Fonte: Autoria própria

A avaliação da função sensoriomotora e do equilíbrio funcional pela EFM e EEB dos 63 indivíduos após AVC está expressa na Tabela 2. Houve um déficit médio na função sensoriomotora de $12,8 \%$, e de $13,2 \%$ no equilíbrio funcional. O coeficiente de variação (CV) da média nas pontuações totais das escalas foi abaixo de $20 \%$. Dentre os domínios da EFM, foi visto um maior CV na função motora (34,9\%) e menor no domínio dor (6,7\%).

Tabela 2 - Análise da função sensório-motora (Escala de Fugl-Meyer) e do equilíbrio funcional (Escala de equilíbrio de Berg) dos indivíduos após AVC crônico $(n=63)$

\begin{tabular}{lrrrr}
\hline & Mínimo & Máximo & $\begin{array}{c}\text { Média* } \\
\text { (DP) }\end{array}$ & CV (\%) \\
\hline Escala de Fugl- & 102 & 244 & $197(34,8)$ & 17,7 \\
Meyer & 16 & 88 & $43(7,0)$ & 16,3 \\
Mobilidade & 31 & 44 & $43(2,9)$ & 6,7 \\
Dor & 3 & 24 & $21(5,2)$ & 24,8 \\
Sensibilidade & 14 & 95 & $72(25,1)$ & 34,9 \\
Função motora & 3 & 62 & $50(22,1)$ & 44,2 \\
FMMS & 11 & 33 & $26(5,3)$ & 20,4 \\
FMMI & 7 & 14 & $11(1,5)$ & 13,6 \\
Equilíbrio & 32 & 56 & $48,6(5,5)$ & 11,3 \\
Escala de equilíbrio & de Berg & & &
\end{tabular}

$\mathrm{CV}$ = Coeficiente de variação da média; $\mathrm{DP}=$ Desvio padrão; FMMS = Função motora de membros superiores; FMMI = Função motora de membros inferiores; $\mathrm{IQ}=$ Intervalo interquartílico.

$*$ = aritmética.

Fonte: Autoria própria

Na análise de correlação entre as variáveis estudadas, a EEB e a EFM totais mostraram uma correlação positiva fraca $(r=0,3569)$, como pode ser visto no diagrama de dispersão da Figura 1. Quando analisada a correlação entre EEB e os domínios da EFM, houve: uma correlação positiva desprezível com a FM total $(r=0,159)$ e a FM de membro superior $(r=0,098)$; uma correlação negativa desprezível com a dor $(r=-0,163)$; uma correlação positiva fraca com a mobilidade $(r=0,489)$ e com a FM de membro inferior $(r=0,344)$; e uma correlação positiva moderada com a sensibilidade $(r=0,512)$. As análises de correlação entre as variáveis podem ser observadas na tabela 3 .

Tabela 3 - Coeficiente de correlação de Pearson entre a EEB, EFM e seus domínios ( $n=63$ )

\begin{tabular}{ccccccccc}
\hline & \multicolumn{9}{c}{ EFM } \\
\cline { 2 - 9 } & Total & Mobilidade & Dor & Sensibilidade & Função motora & FMMS & FMMI & Equilíbrio \\
\hline EEB & 0,357 & 0,489 & $-0,163$ & 0,512 & 0,159 & 0,098 & 0,344 & 0,037 \\
\hline
\end{tabular}

EEB: Escala de equilíbrio de Berg; EFM: Escala de Fugl-Meyer; FMMI: Função motora de membro inferior; FMMS: Função motora de membro superior. Fonte: Autoria própria

\section{DISCUSSÃO}

Os resultados do presente estudo mostram que houve uma associação moderada entre a sensibilidade e o equilíbrio funcional de indivíduos hemiparéticos crônicos após AVC. A mobilidade, a dor e a função motora dos membros superiores e inferiores mostraram uma associação que variou de fraca a desprezível com o equilíbrio funcional.

$\mathrm{Na}$ EFM, a avaliação da função motora de membros inferiores é constituída por motricidade reflexa, movimentação sinérgica e coordenação, itens chaves para o controle motor efetivo do membro inferior. No entanto, tais funções não estiveram associadas ao equilíbrio funcional, apresentando uma correlação positiva fraca neste estudo. Oliveira, Cacho e Borges (2006) demonstraram resultados diferentes, mostrando uma correlação positiva moderada entre a EEB e a função motora de membro inferior da EFM em indivíduos crônicos após AVC. No 
entanto, a amostra foi inferior à do presente estudo, com apenas 20 indivíduos, e apresentou uma média da função motora de membros inferiores de 53,35, diferente da média encontrada neste estudo, com os respectivos desvios padrão distintos. Tais diferenças podem justificar a discordância dos resultados. Portanto, a possível associação entre a função motora de membros inferiores e o equilíbrio funcional de indivíduos após AVC deve ser mais investigada.

De forma semelhante ao que foi constatado presente estudo, Miller et al. (2013) mostraram uma correlação negativa fraca entre a dor e o equilíbrio funcional avaliado pela EEB. No entanto, constataram também que quanto menor a dor, maior a autoconfiança na realização das AVD, o que está relacionado à confiança no próprio equilíbrio. Talvez, durante as AVD, o indivíduo após AVC ignore ou subjugue a dor durante o desempenho da tarefa, com o objetivo de evitar quedas, o que pode justificar a possível relação com a autoconfiança. Vale ressaltar que, no presente estudo, houve uma baixa variabilidade na presença de dor, com uma média alta, ou seja, grande parte dos participantes sentiu pouca dor, o que pode ter interferido na análise, não mostrando uma possível associação que pode existir entre o equilíbrio e a presença da dor mais intensa ou mais dispersa. Portanto, parece que a dor tem um potencial de interferir mais na autoconfiança do indivíduo após AVC, relacionado ao seu equilíbrio nas $A V D$, do que no equilíbrio propriamente dito, embora tal associação precise ser mais bem estudada.

Houve uma correlação positiva desprezível entre a função motora de membro superior e o equilíbrio funcional, ou seja, a capacidade de executar movimentos sinérgicos e coordenados com o membro superior não esteve associada à habilidade de manter o equilíbrio durante tarefas do dia a dia. Sandy et al. (2016) mostraram que ajustes posturais alterados em indivíduos após AVC estão acompanhados de déficits no movimento do membro superior, principalmente relacionados ao alcance funcional. No entanto, esse estudo avaliou apenas o alcance funcional, uma das 14 tarefas propostas pela EEB. Demais tarefas podem ter influenciado no resultado. Além disso, por se tratar de indivíduos na fase crônica após AVC, é de se esperar que adaptações na manutenção do equilíbrio ocorram com o tempo, independentemente de como se apresenta a função motora do membro superior.

Déficits no sistema somatossensorial ocorrem em cerca de $11-85 \%$ dos indivíduos após AVC, afetando a sensibilidade ao toque, a propriocepção e a cinestesia, na maioria dos casos (DOYLE et al., 2011). Estudos prévios mostram que alterações de sensibilidade estiveram associadas a déficits no equilíbrio de indivíduos após AVC (OLIVEIRA et al., 2011; TYSON et al., 2006), de modo semelhante ao que ocorreu no presente estudo, em que foi vista uma correlação positiva moderada entre a sensibilidade e o equilíbrio funcional, ou seja, quanto menos déficits de sensibilidade, menos déficits de equilíbrio funcional. Sabe-se que a informação sensorial influencia no planejamento dos movimentos por meio de sucessivas projeções no córtex pré-frontal pré-motor e nas áreas motoras primárias, áreas responsáveis pelo planejamento e pela execução do movimento (ROSSINI et al., 2003). Parece que tais informações sensoriais, tanto proprioceptivas como exteroceptivas, ajudam nos ajustes posturais necessários para a manutenção do equilíbrio, e após o AVC, quando tais alterações ocorrem, o mecanismo de controle do equilíbrio também pode estar prejudicado (OLIVEIRA et al., 2011). Desse modo, é possível que alterações na sensibilidade possam influenciar na manutenção do equilíbrio funcional de indivíduos após AVC.

\section{CONCLUSÃO}

Déficits no equilíbrio funcional estão associados moderadamente às alterações na sensibilidade dos indivíduos hemiparéticos crônicos após AVC. A mobilidade, a dor e a função motora dos membros superiores e inferiores não demostraram uma associação importante com o equilíbrio funcional. Tais resultados precisam ser reforçados por mais estudos, com o objetivo de serem identificadas as possíveis causas para o déficit no equilíbrio funcional e, consequentemente, serem determinados alvos terapêuticos para a reabilitação efetiva do equilíbrio funcional de indivíduos após AVC.

\section{REFERÊNCIAS}

BLUM, L.; KORNER-BITENSKY, N. Usefulness of the berg balance scale in stroke rehabilitation : a systematic review. Phys. ther., New York, v. 88, n. 5, p. 559-566, 2008.

$\mathrm{CHO}, \mathrm{K}$.; LEE, G. Impaired dynamic balance is associated with falling in post-stroke patients. Tohoku j. exp. med., Tokyo, v. 230, n. 4, p. 233 239, 2013.

FEIGIN, V. L.; NORRVING, B.; MENSAH, G. A. Global burden of stroke. Circ. res., Dallas, v. 120, n. 3, p. 439-448, 2017.

FUGL-MEYER, A. R. et al. The post-stroke hemiplegic patient. 1. a method for evaluation of physical performance. Scand. j. rehabil. med., Stockholm, v. 7, n. 1, p. 13-31, 1975

GLADSTONE, D.; DANELLS, C.; BLACK, S. The fugl-meyer assessment of motor recovery after stroke: a critical review of its measurement properties. Neurorehabilitation neural repair, New York, v. 16, n. 3, p. 232-240, 2002.

HAART, M. de et al. Recovery of standing balance in postacute stroke patients: A rehabilitation cohort study. Arch. phys. med. rehabil., Chicago, v. 85, n. 6, p. 886-895, 2004.

KWONG, P. W. H. et al. A structural equation model of the relationship between muscle strength, balance performance, walking endurance and community integration in stroke survivors. Plos ONE, San Francisco, v. 12, n. 10, p. e0185807, 2017.

LAIBLE, M. et al. Association of activity changes in the primary sensory cortex with successful motor rehabilitation of the hand following stroke. Neurorehabilitation neural repair, New York, v. 26, n. 7, p. 881-888, 2012.

MAKI, T. et al. Estudo de Confiabilidade da aplicação da escala de fugl-meyer no Brasil. Rev. bras. fisioter., São Carlos, v. 10, n. 2, p. 177-183, 2006 
MILLER, K. K. et al. Fatigue and pain: relationships with physical performance and patient beliefs after stroke. Top. stroke rehabil., Frederick, v. 20, n. 4, p. 347-355, 2013.

MIYAMOTO, S. T. et al. Brazilian version of the Berg balance scale. Braz. j. med. biol res., Ribeirão Preto, v. 37, n. 9, p. 1411-1421, 2004.

MUKAKA, M. M. Statistics corner: A guide to appropriate use of correlation coefficient in medical research. Malawi Med. J., Malawi, v. 24, n. 3, p. 69-71, 2012.

NUDO, R. J.; MCNEAL, D. Plasticity of cerebral functions. Hand. clin. neurol., Amsterdam, v. 110, p. 13-21, 2013.

OLIVEIRA, C. B. et al. Abnormal sensory integration affects balance control in hemiparetic patients within the first year after stroke. Clinics, [S.I] v. 66, n. 12, p. 2043-2048, 2011.
OLIVEIRA, R. DE; CACHO, E. W. A.; BORGES, G. Post-stroke motor and functional evaluations: a clinical correlation using fugl-meyer assessment scale, berg balance scale and barthel index. Arq. neuropsiquiatr., São Paulo, v. 64, n. 3 B, p. 731-735, 2006.

ROSSINI, P. M. et al. Post-stroke plastic reorganisation in the adult brain. Lancet neurol., London, v. 2, n. 8, p. 493-502, 2003.

SANDY, M. W. et al. Impaired motor preparation and execution during standing reach in people with chronic stroke. Neurosci. lett., Amsterdam, v. 630, p. 38-44, 2016.

STOKES, M. Cash: neurologia para fisioterapeutas. São Paulo: Premier, 2000.

TYSON, S. F. et al. Balance disability after stroke. Phys. Ther., New York, v. 86, n. 1, p. 30-38, 2006.

Submetido em: 19/11/2018

Acesso em: 29/11/2018 